\title{
Translating, the vital element in classes of French as a foreign language
}

\section{[La traduction : un incontournable en classe de FLE]}

\author{
Jacqueline Oven
}

DOI: 10.18355/XL.2018.11.01XL.30

\begin{abstract}
The article presents the position of translating in classes teaching French as a foreign language. The focus is on the importance of gradual approach (with regard to the level of competence and the age of students as well as to the level of opacity of set phrases) when translating is introduced. Translating appears to be an excellent means for illustrating differences between semantic and lexical units, the semantic unit being the essential part in successful learning of a foreign language. Although set phrases, idioms and proverbs are considered to be the most difficult contents in learning foreign languages, and are not taught or avoided at elementary levels, the article, nevertheless, uses set phrases from certain lexical areas (e.g. colours, body parts, animals, fruits, vegetables), which are normally used in elementary level textbooks, to illustrate the gradual approach in introducing translating.
\end{abstract}

Key words: translating, French as a foreign language (FLE), semantic/lexical unit, lexical area, set phrases

\section{Résumé}

La présente contribution se propose de présenter la place incontournable de la traduction en classe de FLE et l'importance d'une sensibilisation progressive (en fonction du niveau et de l'âge des apprenants tout comme du degré d'opacité variable des expressions idiomatiques) au processus de la traduction. La traduction est en effet un excellent moyen pour mettre en évidence la distinction fondamentale entre unité de sens et unité lexicale. Raisonner en termes d'unité de sens et non plus d'unité lexicale est fondamental dans le processus d'acquisition d'une langue étrangère. Bien que les expressions figées ou idiomatiques soient considérées comme étant les plus difficiles à intégrer lors de l'apprentissage d'une langue étrangère, nous y avons recours pour illustrer le processus de sensibilisation à la traduction en les sélectionnant dans des champs lexicaux apparaissant dans les premières unités d'apprentissage du FLE (comme par exemple les couleurs, les parties du corps, les animaux, les aliments, dont les fruits et les légumes).

Mots-clés: traduction, FLE, unité de sens/unité lexicale, champ lexical, expression idiomatique

\section{Introduction}

Pour enseigner ou apprendre une langue étrangère, les moyens ou méthodes à disposition de l'enseignant ou de l'apprenant sont multiples et variés, dont la traduction qui reste néanmoins souvent à l'écart en classe de langue étrangère, a fortiori en classe de FLE. La complexité du processus de traduction explique la réticence des enseignants à vouloir l'introduire dans les cours de FLE, du moins au début et lors des premiers niveaux d'apprentissage. De plus, qui dit traduction, dit recours à la langue maternelle, ce qui était fortement déconseillé dans les méthodes didactiques de ces dernières décennies. Par ailleurs, concernant les apprenants, les débutants et faux-débutants ne pensent pas avoir les connaissances requises pour pouvoir se soumettre à cet exercice compliqué qu'est la traduction. Peut-on donc réconcilier enseignement/apprentissage du FLE et traduction?

XLinguae, Volume 11 Issue 1XL, January 2018, ISSN 1337-8384, eISSN 2453-711X 
Cette contribution se propose de donner des pistes aux enseignants pour redonner une place à part entière à la traduction en classe de FLE, quels que soient l'âge et le niveau des apprenants, pour faire de la traduction un incontournable en classe de FLE.

La traduction est certes un processus compliqué qui peut effectivement effrayer aussi bien l'enseignant que l'apprenant, mais nous nous efforcerons de montrer qu'une sensibilisation progressive et systématique dès le début de l'apprentissage, en fonction évidemment de l'âge et du niveau de l'apprenant, peut démystifier, voire donner un côté ludique à la traduction. À cet effet, nous nous référerons à des champs lexicaux apparaissant dans les premières unités des manuels de FLE et aurons recours aux expressions dites figées ou idiomatiques relevant de ces champs lexicaux, avec une attention spéciale pour les expressions imagées, choix pouvant sembler quelque peu surprenant, voire paradoxal car la sphère du 'figé' et du 'figuré' est généralement relégué à des niveaux d'enseignement plus avancés.

\section{Complexité du processus de traduction}

La traduction est indéniablement un processus complexe. Les quelques réflexions et définitions suivantes, difficilement contestables, en sont de parfaites illustrations.

Dans son ouvrage sur la traduction au titre révélateur et très certainement provocateur Les Belles infidèles quant à l'exercice périlleux que représente le processus de traduction, en l'associant aux notions de beauté et d'infidélité, G. Mounin (1994 : 8) écrit :

«La culture occidentale est marquée par une «longue tradition qui veut que traduire soit impossible " à cause d'une présumée différence irréductible entre les langues. Toute entreprise de traduction porterait alors "préjudice» à l'original et serait nuisible à tout ce qui constitue ce dernier, de son contenu à sa forme, de son sens à sa lettre, etc. »

A. Berman (2001 : 17), lui, surenchérit d'une certaine manière en employant des termes comme mensonge, vérité, trahison et fidélité :

«Parler de traduction, [...] c'est parler du mensonge et de la vérité, de la trahison et de la fidélité. "

Quant à P. Ricoeur (2010: 12), il évoque aussi bien les difficultés du processus de la traduction que le bonheur suscité par cette activité :

"... elle met le langage à l'épreuve du sens, la traduction est affaire de deuil : celui de la perfection, à laquelle on doit préférer la réussite. Et c'est tant mieux car dans ce deuil de la traduction absolue réside le bonheur même de traduire. »

Le processus de traduction implique effectivement le respect de certaines notions-clés relevant surtout du domaine lingusitique telles que :

- unité de traduction / unité de sens,

- unité lexicale vs unité de sens,

- correspondance vs équivalence,

- dimension contextuelle,

- polysémie,

- sens propre vs sens figuré,

- homonymie,

- connotation (méliorative ou dépréciative), 
Ces notions linguistiques reviennent en effet régulièrement dans les réflexions sur le processus de traduction, avec une prépondérance des termes 'unité de sens' et 'unité de traduction'. Citons-en quelques extraits :

Dans son ouvrage La traduction raisonnée, J. Deslile (1993 : 49) qualifie l'unité de traduction de la manière suivante :

«Sur le plan linguistique, c'est le segment d'un énoncé dont les éléments lexicaux concourent à l'expression d'un seul élément de sens. Nous pouvons assimiler l'unité de traduction à des syntagmes, à des locutions, à des expressions figées et à toutes les séquences de longueur variable qu'il faut traiter en bloc au moment de l'interprétation. 》

Par ailleurs, notons que l'unité lexicale et l'unité de sens sont deux notions rarement superposables, ce qui rend la tâche de l'apprenant du FLE, tout comme d'ailleurs de toute autre langue étrangère, difficile déjà lors de l'acquisistion du vocabulaire et donc a fortiori lors du processus de traduction.

Concernant les notions récurrentes de correspondance et d'équivalence dans le domaine de la traduction, nous pouvons lire dans La traduction aujourd'hui (M. Lederer,1994 : 24-25) :

"La mise en correspondance de deux langues est le premier niveau de la traduction; s'agissant de vocables ou de phrases isolés, elle peut être utile dans l'enseignement des langues; s'agissant de termes monoréférentiels, elle est presque toujours indispensable; s'agissant de textes entiers, elle est inopérante. », /.../ «... (dans le cas de l'équivalence), le sens du texte français est le même que celui de la langue étrangère, mais on remarquera que les mots étrangers et français ne se correspondent que rarement. »

Le lien entre équivalence et correspondance y est décrit comme suit :

"L'équivalence est une correspondance inédite. ", ou encore "L'équivalence s'établit entre textes, la correspondance entre des éléments linguistiques, mots, syntagmes, figements ou formes syntaxiques.Toute traduction comporte certes des correspondances entre des termes et des vocables, mais elle ne devient texte que grâce à la création d'équivalences. »

Outre ces notions, quit dit traduction, dit différents procédés de traduction, qui peuvent être répartis en deux grands groupes, la traduction directe et la traduction oblique :

"Il peut arriver que le message de la langue de départ se laisse parfaitement transposer dans le message de la langue d'arrivée. Le message repose soit sur des catégories parallèles, soit sur des conceptions parallèles. /.../ La traduction directe comprend: l'emprunt, le calque, la traduction littérale. La traduction oblique est une traduction qui ne peut pas être littérale. Elle comprend la transposition, la modulation, l'équivalence, l'adaptation. » (J. -P. Vinay, J. Darbelnet, 1958 : 46).

Ces définitions et réflexions relèvent d'un métalangage linguistique et traductologique, difficilement utilisable en classe de FLE, sauf peut-être dans les cursus linguistiques universitaires. Pour l'enseignant, le défi est donc de familiariser ses apprenants avec ces notions-clés de la traduction, de façon à ce qu'ils les intériorisent et intègrent au fur et à mesure de leur apprentissage du FLE. À cet effet, nous proposons une sensibilisation progressive et systématique (en fonction de l'âge et du niveau de l'apprenant) en puisant dans les champs lexicaux figurant aux différents 
niveaux d'apprentissage pour aborder des notions telles que le figement ou le sens figuré, essentielles et incontournables dans le discernement des unités lexicales des unités de sens ou unités de traduction. Pour illustrer cette dichotomie unité lexicale vs unité de sens/unité de traduction, le recours aux expressions figées ou idiomatiques, en passant des collocations aux expressions imagées jusqu'aux dictons ou proverbes, est une source inépuisable pour l'enseignant, ce qui lui permettra justement de rapprocher le processus de la traduction aux apprenants de manière moins théorique.

\section{Sensibilisation des apprenants à la traduction en classe de FLE}

Face à ces notions utilisées par les linguistes et les traductologues, dont les termes sont difficilement exploitables en classe de FLE, surtout en début d'apprentissage, nous proposons une sensibilisation progressive au fur et à mesure de l'enseignement/apprentissage par le biais d'expressions figées ou idiomatiques - en partant des collocations, expressions (imagées ou pas) jusqu'aux dictons et proverbes. Ce choix peut sembler paradoxal car se référant justement à un vocabulaire communément jugé difficile à acquérir lors du processus d'acquisition d'une langue étrangère, dont le FLE.

\subsection{Expressions idiomatiques}

En lisant les définitions des différentes formes d'expressions figées ou idiomatiques, nous pouvons en noter la grande complexité comme le démontrent les exemples de définition suivants :

"Les langues contiennent des expressions qui sont une sorte de patrimoine linguistique. Chaque langue a son registre d'images, qui sont propres à son génie, à sa culture, à la façon de voir les choses. L'expression imagée est propre au langage, et joue de la différence entre la signification et le sens. Pour traduire l'idée des expressions d'une langue à l'autre, il faut comparer et chercher des expressions correspondantes.» (http://www.lautrequotidien.com, Entretien avec Bernard Cerquiglini sur les mystères des expressions imagées)

"Une séquence est figée du point de vue syntaxique quand elle refuse toutes les possibilités de combinaison ou de transformation. Quand elle est figée sémantiquement, son sens est opaque ou non compositionne, le sens ne peut pas être déduit du sens de ses composants. »(G. Gross, 1996 : 154)

"C'est toute forme grammaticale dont le sens ne peut pas être déduit de sa structure en morphèmes et qui n'entre pas dans la constitution d'une forme plus large. » ( G. Gross, $1996: 4$ )

«Ce sont des combinaisons intraduisibles mot à mot.»(A. Rey, $1979: 6$ )

"C'est une unité polylexicale figée. " (G. Perko, 2003: 100). Plus loin, l'auteur propose également une définition des phrasèmes, une des trois sous-catégories des expressions idiomatiques : «Les phrasèmes (aussi appelés locutions, idiotismes, gallicismes, expressions) sont des expressions polylexicales qui connaissent un très haut degré de figement, ce qui veut dire qu'ils n'admettent presque pas de modifications paradigmatiques ni de transformations syntaxiques. » (G. Perko, 2003 : 102-103)

C. Schapira emploie le terme d'idiotisme pour ces expressions pour le qualifier comme suit : 
" L'idiotisme se définit par son sémantisme non compositionnel et opaque. »(C. Schapira, 1999 : 39).

Par ailleurs, l'auteur part des exemple de revenir à ses moutons et donner sa langue au chat pour décrire le processus de traduction à l'aide du métalangage linguistique :

"Un idiotisme est donc en général intraduisible mot à mot, pourtant parfois il peut être interprété de façon compositionnelle également. L'expression revenir à ses moutons a deux lectures possibles: l'une opaque et l'autre transparente. Utilisée métaphoriquement, il s'agit d'une expression imagée qui signifie "revenir au sujet dont on parlait ». La seconde interprétation est celle de "revenir effectivement à ses moutons ». On voit que dans ce cas-là l'expression ordinairement idiomatique est susceptible d'avoir aussi un sens littéral acceptable.

L'expression donner sa langue au chat ne signifie pas qu'une personne a vraiment remis cet organe du goût à l'animal. Un étranger ne peut pas interpréter cette locution littéralement même s'il connaît le sens habituel de tous les mots qui la composent. On dit que cette phrase n'a pas de lecture compositionnelle et qu'elle $n$ 'est pas transparente, mais plutôt opaque. L'interprétation correcte serait celle-ci: donner sa langue au chat signifie abandonner une réflexion ou s'avouer incapable de répondre à une question. Ainsi on peut dire que si un étranger traduisait cette expression mot à mot, il se rendrait compte de l'opacité sémantique, car la phrase traduite au pied de la lettre serait incompréhensible. Pour cela, il est important de savoir quand il s'agit du sens compositionnel et quand du sens opaque. Celui, qui n'a encore jamais entendu certaines expressions idiomatiques et n'est pas sûr de son sens, doit la chercher dans le dictionnaire et ensuite la traduire dans une langue étrangère de trois manières possibles. »(C. Schapira, 1999 : 38)

En conclusion, les ouvrages traitant des expressions idiomatiques reviennent à développer les mêmes caractéristiques, telles que l'opacité sémantique, la limitation paradigmatique et l'irrégularité morphologique ou syntaxique de la séquence. Certains ouvrages évoquent d'autres propriétés spécifiques aux expressions figées, telles que :

- traces de la langue ancienne (être la lanterne rouge: la lanterne est un objet qui appartient au passé, mais l'expression est toujours présente),

- non-actualisation d'un élément (donner carte blanche: il n'est pas possible d'y ajouter par exemple un adjectif *donner une belle carte blanche, qui n'aurait plus le statut d'expression figée),

- caractère non référentiel des unités lexicales (avoir le sang bleu - le sang et bleu n'ont plus de valeur référentielle ou dénotative),

- non-respect des restrictions de sélection (histoire cousue de fil blanc - l'histoire ne peut pas être cousue),

- impossibilité de traduire littéralement les expressions figées dans une autre langue (mettre les pieds dans le plat).

\subsection{Cohabitation de la traduction et des expressions idiomatiques en classe de FLE}

Suite aux définitions sur la complexité du processus de traduction figurant dans la partie 1. de notre contribution et aux descriptions complexes des expressions idiomatiques, la question sur la pertinence du choix des expressions idiomatiques pour 
familiariser les apprenants au processus de la traduction lors de l'apprentissage du FLE n'est non seulement justifiée, mais s'impose.

Outre le fait que les expressions représentent un excellent moyen de focaliser sur une des notions-clés, sinon la plus pertinente, du processus de traduction - unité de sens (vs unité lexicale), segment incontournable dans l'unité de traduction, nous justifions notre choix en constatant que les expressions idiomatiques incluent souvent des termes relevant des champs lexicaux basiques, figurant dès les premieres unités des manuels FLE.

Les expressions idiomatiques peuvent utiliser du vocabulaire appartenant à différents domaines sémantiques : les animaux, les aliments, etc. La création d'expressions idiomatiques, dans une langue, porte généralement sur des domaines significatifs de l'activité ou des préoccupations des locuteurs de cette langue. Il y a certains domaines qui sont privilégiés pour les expressions les plus fréquentes :

- expressions idiomatiques animalières (expressions renvoyant aux animaux ou à des choses relatives aux animaux),

- expressions idiomatiques botaniques (expressions en rapport avec les végétaux),

- expressions idiomatiques chromatiques (expressions utilisant les noms des couleurs), - expressions idiomatiques corporelles (expressions utilisant une partie du corps humain),

- expressions idiomatiques gastronomiques (expressions utilisant les termes liés aux aliments),

- expressions idiomatiques numériques (expressions utilisant des nombres),

- expressions idiomatiques toponymiques (expressions utilisant des noms de lieux ou de nationalités),

- expressions idiomatiques vestimentaires (expressions un vocaulaire lié aux vêtements).

Par ailleurs, il nous semble extrêmement important, voir impératif d'approfondir la notion d'opacité mentionnée de manière récurrente par les auteurs traitant de ce sujet, caractérisant certes les expressions idiomatiques, mais à laquelle il faudrait, selon nous, ajouter son caractère variable, variabilité dépendant fortement de la langue maternelle des apprenants. Outre le niveau de FLE de l'apprenant et son âge, le degré d'opacité ou la capacité de décrypter le sens d'une expression idiomatique ne sera, selon nous, pas le même selon la langue maternelle employée par les apprenants, qui aura une grande incidence sur le degré d'opacité des différentes expressions idiomatiques. Outre le degré d'opacité inhérente aux expressions idiomatiques françaises, il faudrait prendre en ligne de compte le degré d'opacité apporté par la langue maternelle des apprenants. Par exemple, des expressions telles que baisser les bras, mettre sa main au feu ou rouge comme une tomate pourront être plus ou moins facilement décryptées selon le contexte socio-culturel de l'apprenant et sa langue maternelle. Le degré d'opacité pourra ainsi être plus ou moins élevé si la langue maternelle est l'anglais, le russe ou le chinois. Le degré d'opacité pouvant ainsi être très aléatoire d'une langue maternelle à l'autre, c'est l'enseignant qui devra en évaluer le niveau de difficulté. Cette évaluation lui permettra alors d'intégrer progressivement les différentes expressions idiomatiques en classe de FLE aussi bien qualitativement (en fonction du degré d'opacité) que quantitativement.

Pour résumer, les tableaux 1 et 2 résument nos réflexions sur les expressions idiomatiques et la notion d'opacité dans les expressions idiomatiques : 


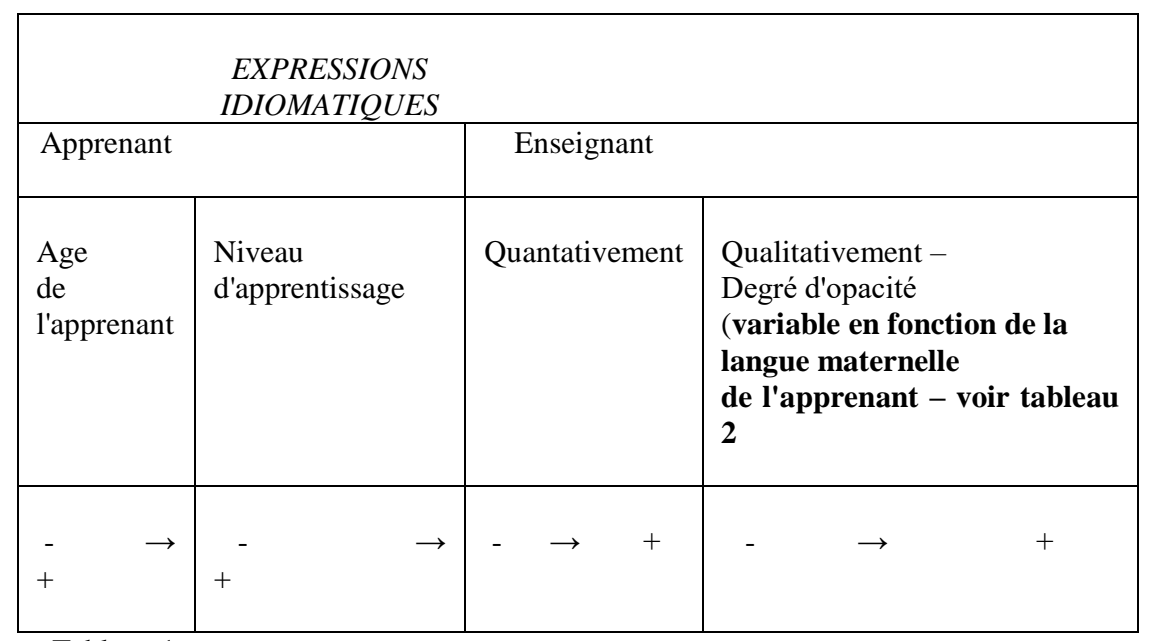

Tableau 1

\begin{tabular}{|l|l|}
\hline $\begin{array}{l}\text { DEGRE D'OPACITE DES EXPRESSIONS } \\
\text { IDIOMATIQUES }\end{array}$ & $\mathbf{O p}=\mathbf{O p 1}+\mathbf{O p 2}$ \\
\hline $\begin{array}{l}\text { Opacité fixe inhérente aux expressions idiomatiques } \\
\text { françaises (O1) }\end{array}$ & $\begin{array}{l}\text { Opacité variable régie par la } \\
\text { langue } \\
\text { maternelle de l'apprenant } \\
(\mathrm{O} 2)\end{array}$ \\
\hline
\end{tabular}

Tableau 2

Enfin, notre expérience nous a montré que le recours aux expressions idiomatiques attire l'attention des apprenants qui comparent, généralement avec curiosité et amusement, ces expressions à celles de leur langue maternelle. Cette approche contrastive est stimulante pour les apprenants, quelles qu'en soient d'ailleurs les constatations (qu'il s'agisse de similitudes, de divergences ou d'interférences. Pour ce faire, l'enseignant peut utiliser tous les champs lexicaux présents dans les manuels de FLE et abordés en classe avec les apprenants, même ceux proposés en début d'apprentissage du FLE. Dans la présente contribution, nous avons ainsi sélectionné des champs lexicaux dits basiques, comme celui des couleurs, des parties du corps, des animaux et des aliments, dont les fruits et légumes.

\subsection{Champs lexicaux des expressions idiomatiques}

Cette partie se propose de présenter quelques champs lexicaux prolifiques et propices à la sensibilisation des apprenants aux notions essentielles d'unité de sens ou d'unité de traduction, fondamentales dans le processus de la traduction (et d'ailleurs dans le degré de maîtrise du FLE et de toute autrelangue étrangère). La liste des expressions idiomatiques présentées dans la suite de notre contribution n'a pas la prétention d'être exhaustive. Nous laissons le soin aux enseignants de la compléter en consultant par 
exemple les ouvrages cités dans notre bibliographie (Bouquet des expressions imagées, Dictionnaire des expressions et locutions, Richesse du vocabulaire, Vocabulaire expliqué du français), en adaptant le degré d'opacité en fonction de la langue maternelle et du contexte socio-culturel de leurs apprenants.

\subsubsection{Champ lexical des couleurs}

Pour introduire le champ lexical des couleurs, nous avons choisi un extrait de l'ouvrage Le petit livre des couleurs rédigé par M. Pastoureau (2005 : 7) qui, au-delà de considérations sur la signification des couleurs, y inclut également quelques expressions idiomatiques :

"Ce n'est pas un hasard si nous voyons rouge, rions jaune, devenons vert de peur, bleu de colère ou blanc comme un linge. Les couleurs ne sont pas anodines. Elles véhiculent des tabous, des préjugés auxquels nous obéissons sans le savoir, elles possèdent des sens cachés qui influencent notre environnement, nos comportements, notre langage, notre imaginaire. Les couleurs ont une histoire mouvementée qui raconte l'évolution des mentalités.»

Nous suggérons ainsi à l'enseignant d'introduire également, quand sont abordées les couleurs, des expressions imagées renfermant des couleurs (en laissant à son appréciation le nombre et le choix de celles-ci). Pourquoi ne pas parler de 'nuit blanche' ou de 'avoir la main verte' quand il s'agit de faire apprendre aux élèves les différents noms de couleur? Cela représente un moyen pour aborder la notion d'unité sémantique ou d'unité de traduction sans devoir utiliser des termes relevant du métalangage linguistique.

Voici un échantillon d'expressions idiomatiques susceptibles d'être utilisées en classe de FLE : annoncer la couleur (faire connaître clairement ses intentions), en voir de toutes les couleurs (subir des épreuves difficiles), un bulletin blanc (bulletin de vote non rempli), un examen blanc (examen non officiel), blanc comme neige (pur, innocent), passer une nuit blanche (passer une nuit sans dormir), faire chou blanc (échouer), un bleu (un débutant), un cordon-bleu (quelqu'un qui fait très bien la cuisine), les Bleus (les joueurs français de football), le sang bleu (sang noble), une peur bleue (une grande peur), être gris (être légèrement ivre), faire grise mine à quelqu'un (lui réserver un mauvais accueil), rire jaune (faire semblant de rire), le maillot jaune (leader du Tour de France), broyer du noir (être déprimé), le marché noir (trafic clandestin et illégal), travailler au noir (travailler sans être déclaré), voir rouge (se mettre très en colère), être dans le rouge (ne plus avoir d'argent sur son compte bancaire), avoir la main verte (avoir un don pour les plantes) ... .

Les expressions idiomatiques renfermant des couleurs peuvent aussi faire l'objet de travaux plus approfondis dans l'enseignement supérieur, sous forme de mémoire de fin d'études avec un métalagange approprié relevant aussi bien de linguistique que de traduction, dont celui de T. Dobnikar intitulé Les couleurs dans les expressions imagées et proverbes français et leurs équivalents en langue slovène. Ce mémoire renferme aussi bien des considérations socio-culturelles sur les représentations ou associations liées aux couleurs dans les deux langues qu'un tableau très complet des expressions idiomatiques françaises contenant une couleur et leurs équivalents slovènes par couleur tout en analysant les procédés de traduction présents lors du passage de la langue source à la langue cible (tels que définis par Vinay et Darbelnet). Les recherches contrastives effectuées dans ce travail amènent des conclusions intéressantes, voire inattendues : les expressions avec couleurs sont plus fréquentes en français qu'en slovène ; le blanc est la couleur qui domine en français, alors que le 
noir, lui, occupe la première place en slovène ; parmi les procédés de traduction, les calques sont très présents, ce qui est plutôt inattendu, vu que les deux langues appartiennent à des groupes de langues différents (langues romanes et langues slaves).

\subsubsection{Champ lexical des parties du corps}

Les expressions renfermant des parties du corps sont extrêmement nombreuses et l'enseignant ne peut qu'être confronté à l'embarras du choix. L'ajout de certaines expressions imagées a pour but surtout de sensibiliser les apprenants à raisonner en termes d'unités de sens et non plus en termes d'unités lexicales, distinction primordiale et incontournable dans le processus de la traduction (quel que soit d'ailleurs le type de texte et son niveau de difficulté). Ce champ lexical est incontournable quand il s'agit de décrire le caractère ou le comportement d'une personne.

Nous proposons quelques expressions exploitables en classe de FLE et que l'enseignant pourrait intégrer à ses cours (dont le choix pourra varier d'un enseignant à l'autre en fonction de la langue maternelle des apprenants, du contexte social et culturel, etc.) : avoir le coeur sur la main (étre généreux), baisser les bras (renoncer à un projet), avoir quelqu'un à l'oeil (le surveiller étroitement), fermer les yeux sur qqch (faire semblant de ne pas voir certaines choses), avoir un poil dans la main (être paresseux), avoir un pied dans la tombe (être très vieux), tourner sept fois sa langue dans sa bouche avant de parler (réfléchir longuement avant de parler), faire la sourde oreille (ne pas vouloir entendre), quand les poules auront des dents (jamais), avoir le bras long (avoir de l'influence), avoir les dents longues (être ambitieux), avoir la langue bien pendue (être bavard), être mauvaise langue (dire du mal des autres), avoir la grosse tête (être hautain), couper les cheveux en quatre (compliquer inutilement les choses), mettre les pieds dans le plat (commettre une maladresse de comportement), ne pas avoir froid aux yeux (agir avec audace), avoir le coeur gros (être triste), avoir une dent contre quelqu'un (lui en vouloir fortement), ... .

Dans son mémoire de fin d'études intitulé Les parties du corps dans les expressions idiomatiques françaises et leurs équivalents dans la langue slovène. Repérage des expressions avec les parties du visage (langue, nez, oeil/yeux, oreille), M. Borštnik (2011) dresse un tableau exhaustif de ces expressions idiomatiques en français auxquelles sont associés les équivalents slovènes, en distinguant les équivalents figés des équivalents non-figés, tout en analysant les équivalents selon la classification proposée par Vinay et Darbelnet. Les équivalents figés sous forme d'expression imagées sont regroupés en quatre sous-groupes : expressions imagées avec les mêmes parties du visage, avec d'autres parties du visage, avec d'autres parties du corps humain et avec d'autres éléments n'appartenant pas au corps humain.

Dans un autre mémoire de fin d'études intitulé Les expressions idiomatiques avec une partie du corps humain, exprimant un trait de caractère ou le comportement d'une personne, dans les langues française, slovène et russe, M. Perko (2012) répertorie d'abord les expressions idiomatiques françaises, slovènes et russes renfermant des parties du corps humain et renvoyant à des traits de caractère ou comportements humains, pour ensuite dresser un tableau avec des équivalents appropriés dans chacune des langues, faisant état aussi bien des similitudes que des divergences tant au niveau de l'analyse des procédés de traduction observés qu'à celui de l'interprétation sémantique de ces équivalents.

XLinguae, Volume 11 Issue 1XL, January 2018, ISSN 1337-8384, eISSN 2453-711X 


\subsubsection{Champ lexical des aliments}

Outre les possibilités mentionnées dans les parties 2.1. et 2.2., les aliments, dont les fruits et légumes, constituent un vivier impressionnant qui permet de sensibiliser entre autres les apprenants aux catégories linguistiques animé vs non-animé ou sens propre vs sens figuré.

De plus, les fruits et légumes ont des connotations généralement dépréciatives quand ils sont employés au sens figuré pour désigner une personne, comme par exemple : une poire ou une pomme (désigne une personne naïve), une courge (désigne une personne bête), une asperge (désigne une personne grande et maigre), un navet (désigne un mauvais film), une patate (désigne une personne grosse), une vache à lait et la poule aux oeufs d'or (une personne qu'on exploite), être soupe au lait (se mettre facilement en colère), .... .

Les expressions imagées suivantes sont susceptibles d'être utilisées en classe par l'enseignant, qui aura préalablement établi le degré d'opacité en fonction de la langue maternelle de ses apprenants : tomber dans les pommes (s'évanouir), travailler pour des prunes (travailler pour rien), mettre du beurre dans ses épinards (améliorer sa situation financière), avoir un oeil au beurre noir (avoir un oeil noir suite à un coup), la cerise sur le gâteau (petit détail qui couronne une action), couper la poire en deux (trouver un compromis), avoir un coeur d'artichaut (personne infidèle), mettre de l'eau dans son vin (modérer ses exigences), avoir du pain sur la planche (avoir beaucoup de travail à faire), faire le poireau (attendre), raconter des salades (raconter des choses qui ne sont pas vraies), mettre son grain de sel (ajouter son avis lors d'une conversation sans y être sollicité), tourner au vinaigre (situation qui tourne mal), être comme un coq en pâte (être heureux), bon comme le pain (très bon), long comme un jour sans pain (très long)... .

\subsubsection{Champ lexical des animaux}

Le champ lexical des animaux est, lui aussi, une source inépuisable pour l'enseignant de FLE qui voudrait familiariser l'apprenant avec des notions-clés intervenant lors du processus de traduction. Il serait également intéressant de demander à l'apprenant de proposer pour ces expressions des équivalents dans sa langue maternelle, ce qui lui permettrait de s'initier à comparer, relativiser et à intégrer les critères d'idiomatie et de fréquence dans son processus d'apprentissage, levier indispensable pour pouvoir abandonner peu à peu un raisonnement en termes d'unités lexicales.

Quelques exemples pour illustrer la catégorie animé vs non-animé et celle de connotation (généralement dépréciative) : une vache (quelqu'un de méchant), un porc (quelqu'un ayant un comportement inadmissible), un âne (quelqu'un de bête), une bécasse (une femme pas très intelligente), un asticot (quelqu'un de grand et maigre), un pigeon (quelqu'un de naîf), un poulet (un policier), un mouton (quelqu'un n'ayant aucune personnalité et suivant les autres), un corbeau (auteur de lettres anonymes), une mère poule (possessive envers ses enfants), une langue de vipère (quelqu'un qui dit du mal des autres), une fine mouche (quelqu'un d'habile et de rusé), un ours mal léché (quelqu'un de désagréable et de peu sociable), un chaud lapin (homme porté sur les plaisirs de l'amour), .... .

D'un point de vue contrastif, les expressions avec 'comme' peuvent représenter une piste intéressante en classe de FLE : être rusé comme..., être bavard comme..., être doux comme..., être muet comme..., être rouge comme..., être têtu comme..., être laid/moche comme..., être myope comme..., dormir comme... . L'exercice est d'autant 
plus pertinent si la langue maternelle des apprenants connait cette même structure permettant d'exprimer l'idée de 'beaucoup', 'très', 'bien' ou 'mal', avec généralement des éléments de comparaison différents de ceux figurant dans les expressions françaises, ce qui les amène à comparer, élément non sans importance en classe, et à intérioriser la notion de différence, sans la considérer forcément comme une source de difficulté ou d'obstacle dans leur processus d'apprentissage.

D'autres expressions ou proverbes susceptibles d'être introduits en classe de FLE : s'entendre comme chien et chat (ne pas s'entendre avec quelqu'un), mener une vie de chien (mener une vie difficile), appeler un chat un chat (appeler les choses par leur nom), avoir un chat dans la gorge (être enroué), avoir d'autres chats à fouetter (avoir d'autres choses plus importantes à faire), poser un lapin (ne pas venir à un rendezvous), se jeter dans la gueule du loup (aller au-devant des dangers), prendre le taureau par les cornes (affronter une difficulté), avoir des oursins dans les poches (être radin, avare), avoir le cafard (ne pas avoir le moral), être comme un éléphant dans un magasin de porcelaine (se comporter de façon très maladroite), sauter du coq à l'âne (parler de manière confuse, décousue), monter sur ses grands chevaux (se mettre en colère), être comme un coq en pâte (se sentir bien), avoir une mémoire d'éléphant (avoir une excellente mémoire) ; II n'y a pas un chat (il n'y a personne) ; La nuit, tous les chats sont gris (choses ou personnes difficilement différenciables) ; Chat échaudé craint l'eau froide (craindre une mauvaise expérience déjà vécue), ... .

Dans son mémoire de fin d'études intitulé Les animaux dans les expressions idiomatiques françaises et leurs équivalents dans les langues anglaise et slovène, Špela Mencin (2010) répertorie les expressions idiomatiques françaises et leurs équivalents en anglais et en slovène, tout en indiquant pour chaque expression française un équivalent non figé en français, de manière à mettre en évidence le sens attribué à chacune de ces expressions et donc l'unité de sens ou l'unité de traduction, une des clés pour une acquisition efficace de toute langue étrangère. Un des exemples figurant dans ce mémoire est appeler un chat un chat, pour lequel l'auteure propose tout d'abord un équivalent non-figé (parler franchement) pour ensuite déterminer son équivalent en anglais (avec ou sans animal) to call a spade a spade et en slovène (avec ou sans animal) reci bobu bob in popu pop. Lors du repérage de ces expressions dans les trois langues mentionnées, il ressort que ce sont les animaux domestiques qui sont le plus fréquemment représentés et utilisés dans les trois langues.

\section{Conclusion}

La traduction est un élément essentiel dans l'apprentissage de toute langue étrangère, a fortiori du FLE, et devrait être intégrée dans le processus d'apprentissage dès ses débuts. Le processus de traduction exige en effet une délimitation nette entre l'unité de sens et l'unité lexicale, élément déterminant dans l'acquisition réussie d'une langue étrangère. Il est donc nécessaire de familiariser l'apprenant au processus de traduction en classe de FLE, tout en procédant évidemment à une sensibilisation progressive en fonction du niveau et de l'âge de l'apprenant tout comme du degré d'opacité des expressions idiomatiques, pouvant sensiblement varier d'une langue maternelle à une autre, fait dont l'enseignant devrait absolument tenir compte en classe de FLE. Les notions-clés en matière de traduction peuvent en effet être présentées aux apprenants non pas en ayant recours au métalangage linguistique, mais en utilisant les expressions idiomatiques comme par exemple les collocations, expressions (imagées), dictons ou proverbes, qui représentent un vivier inépuisable pour l'enseignant, qui peut y trouver des outils pour mettre en oeuvre un apprentissage centré sur les unités de sens, garant d'une bonne acquisition du FLE. A cet effet, même les champs lexicaux les plus basiques, apparaissant dans les premières unités des manuels, tels

XLinguae, Volume 11 Issue 1XL, January 2018, ISSN 1337-8384, eISSN 2453-711X 
que les couleurs, les parties du corps, les animaux et les aliments, dont les fruits et légumes, permettent à l'enseignant de procéder à une sensibilisation progressive de l'apprenant aux unités de sens. Raisonner en termes de sens à partir d'expressions idiomatiques, au sens large du terme, au-delà des considérations focalisées sur la notion de sens, donne également l'occasion à l'apprenant d'avoir recours à sa langue maternelle, à comparer, à relativiser, à s'ouvrir plus facilement au monde extérieur, à lui donner l'envie de découvrir, sans que pour autant les divergences ne soient un obstacle dans ce cheminement.

\section{Bibliographic references}

BERMAN, A. 2001. Au début était le traducteur. In : TTR traduction, terminologie, rédaction, 14. 2. 2001. ISSN 1708-2188 (numérique) 0835-8443 (imprimé)

BOCHART-FIEVEZ, J. - DELAHAUT, J. 1994. Richesse du vocabulaire, tome I. Louvain-la-Neuve : Editions Duculot. ISBN 2-8011-0879-0

BOCHART-FIEVEZ, J. - DELAHAUT, J. 1992. Richesse du vocabulaire, tome II. Paris - Louvain-la-Neuve : Editions Duculot. ISBN 2-8011-1020-5

BOCHART-FIEVEZ, J. 1996. Richesse du vocabulaire, tome III. Louvain-la-Neuve : Editions Duculot. ISBN 2-8011-1081-7

BORŠTNIK, M. 2011. Les parties du corps dans les expressions idiomatiques françaises et leurs équivalents dans la langue slovène (mémoire de fin d'études, sous la direction de V. Pogačnik et J. Oven). Ljubljana : Filozofska fakulteta

DELISLE, J. 1993. La traduction raisonnée. Ottawa : Presses universitaires. ISBN 27603-0372-1

DOBNIKAR, T. 2011. Les couleurs dans les expressions imagées et proverbes français et leurs équivalents en langue slovène (mémoire de fin d'études, sous la direction de V. Pogačnik et J. Oven). Ljubljana : Filozofska fakulteta

DUNETON, C. 1990. Le Bouquet des expressions imagées. Paris: Seuil. ISBN 9782020099585

GROSS, G. 1996. Les expressions figées en français - noms composés et autres locutions. Paris : Editions Ophrys. ISBN 9782708007895

KLEIN, J.-R. 2010. Les expressions verbales figées de la francophonie, Paris : Ophrys, Paris. ISBN 9782708012387

LADMIRAL, J.-R. 1994. Traduire : Théorèmes pour la traduction. Paris : Gallimard. ISBN 13: 9782070737437

LARGER, N. - MIMRAN, R. 2004. Vocabulaire expliqué du français. Paris : CLE International. ISBN 2-09033719-2

LEDERER, M. 1994. La traduction aujourd'hui : le modèle interprétatif. Paris : Hachette. ISBN 9782011550002

LEDERER, M. 2009. Le sens dessus dessous: herméneutique et traduction. In : Cercel, Larisa, dir. : Übersetzung und Hermeneutik - Traduction et herméneutique, Bucarest (Zêta Books). ISSN 0026-0452 (imprimé) 1492-1421 (numérique)

MENCIN, S. 2010. Les animaux dans les expressions idiomatiques françaises et leurs équivalents dans les langues anglaise et slovène (mémoire de fin d'études, sous la direction de V. Pogačnik et J. Oven). Ljubljana : Filozofska fakulteta

MOUNIN, G. 1994. Les belles infidèles. Paris : Presses Universitaires de Lille. ISBN 978-2-85939-459-2

PASTOUREAU, M. 2005. Le petit livre des couleurs. Paris : Éditions Points. ISBN $13: 978-2755700343$

PERKO, G. 2001. Les expressions idiomatiques: description théorique et traitement dictionnairique (l'exemple des dictionnaires monolingues français). In : Linguistica XLI, 37-75. Ljubljana : Filozofska fakulteta. ISSN 0024-3922 (imprimé) 2350-420X (numérique) 
PERKO, G. 2003. Les expressions idiomatiques: Un mal nécessaire? Actes du $2^{\text {ème }}$ Colloque sur les études françaises en Croatie, 99-112. Zagreb: ArTresor. ISSN 23194466

PERKO, M. 2012. Les expressions idiomatiques avec une partie du corps humain, exprimant un trait de caractère ou le comportement d'une personne, dans les langues française, slovène et russe (mémoire de fin d'études, sous la direction de V. Pogačnik et J. Oven). Ljubljana : Filozofska fakulteta

REY, A. 1989. Dictionnaire des expressions et locutions. Paris : Robert (Usuels). ISBN 9782850361036

RICOEUR, P. 2010. Sur la traduction. Paris : Bayard. ISBN 9782227473676

SCHAPIRA, C. 1999. Les stéréotypes en français: proverbes et autres formules. Paris : Editions Ophrys. ISBN 2708009117

VINAY, J.P. - DARBELNET, J. 1977. Stylistique comparée du français et de l'anglais. Paris : Didier. ISBN 9782278008940

(http://www.lautrequotidien.com, Entretien avec Bernard Cerquiglini sur les mystères des expressions imagées)

Words: 6127

Characters: 40931 (22,74 standard pages)

Dr. Jacqueline Oven

Faculty of Arts

University of Ljubljana

Aškerčeva 2, 1000 Ljubljana

Slovenia

jacqueline.oven@ff.uni-lj.si 